\title{
Consideraciones en cuanto al uso medicinal y recreacional de la marihuana y sus efectos sobre el pulmón
}

\author{
GONZALO ALVEAR T.*
}

\begin{abstract}
Reflections about medicinal and recreational use of marijuana and its effects on the respiratory system
\end{abstract}

Marijuana use has increased in Chile in recent years, particularly in teenagers and young people. It seems to be a general perception that its use does not involve great risks. The current discussion on the legalization of marijuana should make the necessary distinction between medical and recreational use of it. There are few indications approved by international health agencies for medicinal use, all of them with synthetic derivatives and oral administration, and countless other indications based mostly on studies with serious methodological flaws. The recreational use of marijuana, in addition to the widely known psychosocial deleterious effects, induce on the respiratory system the onset of chronic respiratory symptoms, airway inflammation and immunomodulatory effects. Depending on the exposure time and amount, use of marijuana has been associated with adverse effects on pulmonary function, development of COPD and lung cancer. Therefore, the recreational use without restriction of this drug could have adverse impact on personal and public health, so then the free use of marijuana should not be recommended.

Key words: Marijuana, cannabis, medical use, recreational use, respiratory adverse effects.

\section{Resumen}

El consumo de marihuana ha aumentado en Chile en los últimos años, especialmente en jóvenes y adolescentes y pareciera existir una percepción general de que su uso no conlleva mayores riesgos. La discusión actual sobre la legalización de la marihuana debe hacer la distinción necesaria entre uso medicinal y uso recreacional de ella. Existen escasas indicaciones aprobadas por los organismos de salud internacionales para su uso medicinal, todas ellas con derivados sintéticos de administración oral, y un sinnúmero de otras indicaciones basadas en su gran mayoría en estudios que adolecen de serias deficiencias metodológicas. El uso recreacional de marihuana, además de los efectos psicosociales deletéreos ampliamente conocidos, induce sobre el sistema respiratorio la aparición de síntomas crónicos de la vía aérea, inflamación local y efectos inmunomoduladores y, dependiendo del tiempo y cantidad de exposición, se ha asociado con efectos negativos sobre la función pulmonar, desarrollo de EPOC y de cáncer pulmonar. Por tanto, el uso recreacional sin restricción de esta droga podría tener a futuro gran impacto desfavorable sobre la salud personal y la salud pública, por lo que no debería recomendarse el consumo libre de la marihuana.

Palabras clave: Marihuana, cannabis, efectos medicinales, uso recreacional, efectos adversos respiratorios.

* Clínica Miguel de Servet e Integramédica. 


\section{Introducción}

La Cannabis sativa, cultivada y producida prácticamente en todos los países del orbe, es hoy en día la droga ilícita más consumida a nivel mundial. Se estima que 180,6 millones de personas en el mundo consumieron marihuana durante 2011, lo que equivale al 3,9\% de la población total adulta entre los 15-64 años ${ }^{1}$. En Chile, el Décimo Primer Estudio Nacional de Drogas en Población General mostró, entre los años 2012 y 2014 , un aumento en la prevalencia de consumo anual de marihuana (desde 7,1 el 2012 a 11,3 el 2014, independiente de la edad, sexo y nivel socioeconómico), un aumento significativo de la tasa de incidencia en jóvenes (de $2,7 \%$ a $7,5 \%$ ) y adolescentes $(3,3 \%$ a $5,5 \%)$ y un aumento significativo en la prevalencia de uso problemático de marihuana $(1,8 \% \text { a } 2,5 \%)^{2}$.

Se considera una sustancia "ilícita" desde 1970, cuando se clasificó como una droga de tipo I, es decir, perteneciente al grupo de drogas, sustancias o químicos sin uso médico aceptado $\mathrm{y}$ de alto riesgo potencial de abuso ${ }^{3}$. A pesar que esta clasificación aún se mantiene al día de hoy, pareciera existir cada vez más una percepción en la población general en cuanto a que el consumo de marihuana no conlleva ningún daño para la sa$\operatorname{lud}^{2,4}$, por lo que su acceso no debería ser regulado ni menos prohibido ${ }^{5}$. Esta percepción podría estar "contaminada" por la cada vez más frecuente aparición en los medios de prensa de los potenciales efectos beneficiosos medicinales de esta planta, el creciente número de estados que han legislado a favor del uso médico de la cannabis y el intenso lobby mediático "a favor" de ella. Pero lo que más llama la atención es la legalización de la producción y venta regulada para consumo no medicinal ya no sólo en un país (Uruguay), sino que en dos Estados de EE.UU. (Colorado y Washington) ${ }^{6}$, sin la existencia de evidencia sobre la seguridad de su uso a largo plazo.

El uso medicinal de la marihuana tiene como fin tratar alguna enfermedad o aliviar los síntomas, por lo que se debe hacer la distinción entre el uso medicinal versus el uso recreacional de la marihuana, lo que lleva implícito también la distinción necesaria entre la legalización del uso medicinal versus la legalización del uso recreacional.

En este artículo se presentarán los potenciales usos medicinales de la marihuana, revisando sus probables efectos beneficiosos y adversos derivados de su consumo, enfocándose específicamente en los efectos de esta droga sobre el sistema respiratorio.

\section{Uso medicinal de la marihuana}

La marihuana proviene de la planta Cannabis sativa, que contiene más de 400 compuestos del tipo flavonoides y terpenoides ${ }^{7}$ y sustancias químicas propias llamadas "cannabinoides", de las cuales más de 60 son farmacológicamente activas $^{8}$. Todas ellas tienen en común la capacidad de activar receptores endógenos del tipo $\mathrm{CB} 1$ y CB2, gatillando así sistemas de señalización específicos ${ }^{9}$ que inducen múltiples acciones al inhibir directamente la secreción de varios neurotransmisores, como la acetilcolina, dopamina y glutamato, e indirectamente de otros tantos ${ }^{4}$. Los receptores $\mathrm{CB} 1$ se encuentran principalmente en el ganglio basal, cerebelo, hipocampo, corteza de asociación, médula espinal y nervios periféricos, mientras que los receptores CB2 están en las células del sistema inmune ${ }^{4}$.

Los cannabinoides más conocidos y estudiados son el delta-9-tetrahidrocanabinol (THC), que es el principal componente psicoactivo de la marihuana y el cannabidiol, que tendría efectos ansiolíticos y antipsicóticos ${ }^{10}$.

El THC, cuya fuente principal es la resina de las hojas y del tallo, es altamente liposoluble y se absorbe rápidamente en el aparato respiratorio e intestinal, teniendo una biodisponibilidad al fumarla entre el $15-20 \%$, comparado con un $6 \%$ al ingerirla oralmente ${ }^{11}$.

Los efectos terapéuticos de la marihuana dependen de la concentración del THC y de la relación THC/cannabidiol debido a la capacidad del cannabidiol de mitigar los efectos psicoactivos del $\mathrm{THC}^{12}$, siendo esta relación 1:1 la que provee los mejores beneficios clínicos con los menores efectos adversos ${ }^{13}$. También depende de la vía de administración, ya que esta determina la farmacología, el proceso de absorción y el metabolismo de los distintos cannabinoides ${ }^{14}$.

Los cannabinoides pueden ser administrados vía oral, sublingual, tópica, inhalada o fumada, pudiendo ser extraídos naturalmente de la planta o fabricados sintéticamente. Debido a su alta liposolubilidad, el THC se deposita en los tejidos grasos, siendo liberada lentamente hacia la sangre hasta ser eliminada completamente del organismo en un proceso que puede durar hasta 5 semanas ${ }^{15}$. Es por eso que el ingerir cantidades importantes de marihuana puede mantener sus efectos por un tiempo prolongado.

En la actualidad, la marihuana sólo ha sido aprobada para su uso medicinal frente a escasas situaciones clínicas. Es así como la FDA (Food and Drug Administration, EE.UU) aprobó su uso para controlar las náuseas y los vómitos produci- 
dos por la quimio y radioterapia y para estimular el apetito en pacientes con SIDA y desnutrición. La aprobación está dada para el dronabinol y la nabilona, dos derivados sintéticos de la THC, ambos de uso oral ${ }^{16}$. En el Reino Unido y otros países europeos, por su parte, se aprobó el uso de los nabiximoles para aliviar el dolor neuropático crónico y la espasticidad muscular en la esclerosis múltiple. Su nombre comercial es Sativex ${ }^{\circledR}$ y su forma farmacéutica es una solución oral para pulverizar ${ }^{17}$.

Como todo fármaco, el uso medicinal de la marihuana (y por tanto, su legalización) debería basarse en datos empíricos que aseguren, primero, los beneficios tanto personales como en la salud pública y segundo, la mínima ocurrencia de eventos adversos ${ }^{18}$.

\section{¿Cuán sólidas son las evidencias disponibles que apoyan estas indicaciones y la seguridad de su uso?}

En cuanto al control de las náuseas en enfermos de cáncer, un meta-análisis reciente ${ }^{19}$ mostró que el dronabinol (no así la nabilona) tiene mejores resultados antieméticos que las drogas clásicamente usadas (neurolépticos), aun cuando los autores hacen notar que estos efectos deben ser considerados con precaución debido al pequeño número de pacientes incluidos en los estudios. No obstante, y tomando en cuenta la disponibilidad de potentes fármacos antieméticos que se han desarrollado ${ }^{20}$, los cannabinoides no están recomendados como tratamiento de primera línea para combatir las náuseas en pacientes con cáncer, aunque podrían tener algún rol como tratamiento agregado $^{21}$. En cuanto a su utilidad en pacientes con SIDA, una revisión reciente Cochrane no encontró evidencias que avalen la eficacia ni la seguridad de los cannabinoides en esta patología $^{22}$. En lo que se refiere a la efectividad de los componentes sintéticos de la marihuana para el tratamiento de la espasticidad muscular y el dolor neuropático en la esclerosis múltiple, los estudios no permiten llegar a conclusiones definitivas y cualquier eventual beneficio es probablemente pequeño, mientras que los potenciales eventos adversos son comunes y la seguridad a largo plazo aún no ha sido establecida ${ }^{21}$.

Un aspecto que involucra transversalmente a todas estas indicaciones es la falta de evidencias definitivas sobre las dosis óptimas particulares para cada una de las enfermedades ${ }^{23}$.

A pesar de esta falta de evidencias concluyentes sobre el rol beneficioso de la marihuana para los casos aprobados por los organismos internacionales, 23 estados de EE.UU. (más el distrito de Columbia), Israel, Canadá y Países Bajos han legislado, desde el año 1996, en favor del uso medicinal de la marihuana y permitiendo aún un uso mayor al de las recomendaciones anteriormente mencionadas. Probablemente esto se origina en publicaciones que han evaluado su uso en otras patologías y para el control de síntomas, como el dolor neuropático de origen distinto al de la esclerosis múltiple, el glaucoma, la enfermedad de Crohn, el desorden de estrés postraumático, la epilepsia, la enfermedad de Alzheimer o el dolor crónico en pacientes con cáncer ${ }^{24-37}$. Una limitación habitual de estos estudios es su escaso número de pacientes, su alto riesgo de sesgos $\mathrm{y}$ sus resultados marginales en cuanto a evidenciar escasos beneficios versus un alto riesgo de presentar eventos adversos.

Un aspecto que provoca escepticismo sobre la existencia de algún mecanismo de acción común efectivo de la marihuana en estas enfermedades es justamente el hecho que todas estas condiciones tienen distinta etiología, fisiopatología y fenomenología ${ }^{23}$, por lo que no es científicamente plausible plantear la efectividad de la marihuana en todas ellas.

\section{Uso recreacional de la marihuana}

El uso recreacional de la marihuana difiere diametralmente al potencial uso medicinal de ella dada la disponibilidad de suficiente evidencia clínico-epidemiológica acerca de los efectos deletéreos que produce en el largo plazo el fumar o inhalar marihuana, especialmente en los adolescentes ${ }^{38}$.

Probablemente esto se deba, entre otras razones, a las diferentes formas y cantidades consumidas con ambos tipos de usos.

Los estudios sobre los efectos medicinales de la marihuana se han llevado a cabo con derivados sintéticos orales, con dosis reguladas y fijas del compuesto activo (para los escasos estudios publicados del uso medicinal de la marihuana inhalada, no existen dosis establecidas ${ }^{7}$ ).

Con el uso recreacional, en cambio, el consumo es inhalado o aspirado y de todos los componentes combustibles de la hoja de la planta, sin existir una cantidad definida ni fija de cada uno de ellos. Del mismo modo, en el uso terapéutico están absolutamente definidas la dosis necesarias que deben ser ingeridas diariamente para lograr los efectos beneficiosos esperados, mientras que con el uso recreacional no existen antecedentes en cuanto a las "dosis" diarias potencialmente seguras. De hecho, la "potencia" de los productos 
de la cannabis ha ido aumentando progresivamente debido a las cada vez más sofisticadas formas de cultivo. En la década de los años 6070 , un "pito" de marihuana contenía en promedio $10 \mathrm{mg}$ de THC, cifra que contrasta con cerca de $150 \mathrm{mg}$ que puede alcanzar con las subespecies actuales ${ }^{39}$.

La evidencia en cuanto a los efectos psicosociales deletéreos del consumo de marihuana en forma recreacional es amplia. Se sabe que el fumar marihuana conlleva un riesgo significativo de adicción, especialmente en los adolescentes y en aquellos que la fuman diariamente ${ }^{40}$. También hay evidencias que muestran que impacta en el desarrollo cerebral normal. Los endocannabinoides juegan un rol crítico en el desarrollo y maduración cerebral, especialmente durante la niñez y la adolescencia ${ }^{41}$, ya que estos procesos se mantienen hasta los 21 años en promedio ${ }^{42}$. A diferencia de los endocannabinoides, de una duración de acción corta, los exocannabinoides presentes en la marihuana actúan sobre el sistema de endocannabinoides de forma prolongada, resultando en una activación no fisiológica? Todo esto lleva al desarrollo de alteraciones en la conectividad neuronal (menor cantidad de fibras) en regiones cerebrales específicas como las prefrontales y subcorticales ${ }^{5}$, lo que explicaría los hallazgos que relacionan el uso frecuente de marihuana desde la adolescencia con una disminución significativa del coeficiente intelectual ${ }^{43} \mathrm{O}$ con el peor rendimiento escolar comparado con los que no la fuman ${ }^{44}$.

Otros hallazgos han relacionado el uso temprano y frecuente de la marihuana con mayor riesgo de "escalar" hacia otras drogas ilícitas "más pesadas" y también con el desarrollo de alteraciones mentales como ansiedad y depresión, pero especialmente con mayor riesgo de psicosis, sobre todo en pacientes con vulnerabilidad genética ${ }^{45} \mathrm{y}$ con esquizofrenia ${ }^{5}$, aunque en todos estos últimos casos, el establecimiento claro de un rol causal de la marihuana es prácticamente imposible de demostrar debido a la coexistencia de muchos otros factores tanto psicosociales como ambientales.

Se han descrito también efectos adversos a corto plazo, como alteraciones en la memoria reciente, descoordinación motora y alteraciones del juicio ${ }^{12}$. Una asociación reciente ha vinculado el uso de marihuana con riesgo de accidentes de tránsito fatales ${ }^{46}$, lo que se apoya en el hecho de la relación positiva entre los niveles sanguíneos de THC y la alteración en la capacidad de conducción vehicular ${ }^{47}$.

Por último, estudios preliminares han encontrado asociación entre el consumo de marihuana con infarto al miocardio, accidentes vasculares cerebrales y enfermedad vascular periférica ${ }^{48}$.

\section{Efectos del uso recreacional de la marihuana sobre el sistema respiratorio}

Para comprender mejor los efectos del fumar marihuana sobre el pulmón, es útil su comparación con el modelo de consumo de los cigarrillos en cuanto a la composición del humo resultante de la combustión y con la técnica o modalidad con la cual esta droga se fuma.

El humo resultante de la combustión de la hoja de la marihuana contiene una compleja mezcla de sustancias químicas, siendo algunas de ellas propias de la marihuana (canabinoides) y otras cualitativamente similares a las del humo del tabaco (a excepción de la nicotina $)^{49}$, como son el amonio, el ácido hidrociánico, las nitrosaminas, fenoles, naftaleno, benzopireno, benzantrazeno, etc $^{50}$.

La "técnica" inhalatoria al fumar marihuana difiere de la utilizada por los fumadores de cigarrillos. En el primer caso se hacen inhalaciones más profundas y prolongadas y se fuma en menor cantidad de tiempo y con mayores temperaturas de combustión ${ }^{51}$. Esta técnica inhalatoria resulta en aproximadamente 5 veces mayor concentración de carboxihemoglobina, cuatro veces más cantidad de alquitrán inhalado y la retención de un tercio más de alquitrán en las vías aéreas inferiores en comparación con el consumo de cigarrillos ${ }^{11}$.

\section{Efectos a corto plazo sobre la función pulmonar}

Uno de los efectos inmediatos más conocidos de la inhalación de marihuana es la broncodilatación $^{52}$, reconocido ya desde fines de 1800 , época en que se recomendaba la marihuana como tratamiento para el asma ${ }^{53}$. Los primeros estudios clínicos que demostraron experimentalmente estos efectos broncodilatadores en sujetos jóvenes y sanos fueron publicados el año 1973 en la revista New England Journal of Medicine, aunque el número de participantes analizados fue escaso y uno de los estudios usó una forma no fumada de administrar la marihuana ${ }^{54,55}$. Posteriormente, se demostraron estos mismos efectos en sujetos con asma leve y en pacientes con broncoespasmo inducido por metacolina y ejercicio ${ }^{56}$. Es así que con toda la evidencia disponible hasta hoy en día, se ha logrado establecer que la inhalación de marihuana al fumarla produce una broncodilata- 
ción que alcanza su máximo a los 15 min y dura aproximadamente $1 \mathrm{~h}^{57}$. Un meta-análisis relativamente reciente ${ }^{57}$ que evaluó en 12 estudios los efectos a corto plazo sobre la función pulmonar, mostró que el fumar marihuana indujo en forma aguda un aumento de 0,15 a $0,25 \mathrm{~L}$ en promedio en el volumen espiratorio forzado en el $1^{\text {er }}$ segundo: $\mathrm{VEF}_{1}$ (5 estudios) y un aumento entre el $8 \%$ y $48 \%$ en la conductancia específica (sGaw) (7 estudios). Si bien este meta-análisis demostró efecto broncodilatador agudo al fumar marihuana, metodológicamente tiene limitaciones, siendo una de las principales el no identificar si los sujetos incluidos eran sanos o tenían alguna patología respiratoria tipo asma bronquial. Esto es importante ya que no está claro el mecanismo por el cual se produce la broncodilatación, existiendo estudios en animales que han mostrado que la anandamida, un cannabinoide endógeno que es capaz de activar los receptores CB1 e inhibir la broncoconstricción ${ }^{58}$, ejerce una acción broncodilatadora principalmente cuando la musculatura de la vía aérea está previamente contraída. De lo contrario podría inducir broncoconstricción ${ }^{59}$. El THC imita la acción de la anandamida, pero sus efectos son más potentes y de mayor duración ${ }^{60}$.

\section{Efectos a largo plazo}

\section{a) Función pulmonar}

Múltiples estudios han investigado los efectos a largo plazo de la marihuana fumada sobre la función pulmonar. Un meta-análisis que incluyó 14 estudios no mostró asociación entre el fumar marihuana y la obstrucción de la vía aérea a largo plazo $^{57}$. Un estudio posterior ${ }^{61}$, que comparó los efectos de la marihuana y el tabaco, tampoco mostró disminución significativa del $\mathrm{VEF}_{1}$ ni de $\mathrm{VEF}_{1} / \mathrm{CVF}$ (capacidad vital forzada) en los fumadores de marihuana. Tampoco hubo evidencias del desarrollo de enfisema en TAC de alta resolución, aunque el uso de marihuana se asoció con signos tomográficos sugerentes de hiperinsuflación pulmonar. Un estudio más reciente ${ }^{62}$ tampoco mostró asociación entre fumar marihuana y obstrucción de la vía aérea al controlar el efecto de exposición a tabaco, pero se asoció con mayor CVF, mayor resistencia de la vía aérea (Raw) y menor sGaw. Un estudio de seguimiento a 20 años ${ }^{63}$ mostró que aquellos fumadores de bajas cantidades acumuladas de marihuana (7 pitos/ año de exposición total) presentaban un aumento tanto del $\mathrm{VEF}_{1}$ como de la CVF, pero aquellos con exposición mayor a esto, mostraron una disminución del $\mathrm{VEF}_{1}$ en el tiempo. Si bien este estudio incluyó un porcentaje muy pequeño de fumadores de grandes cantidades de marihuana, lo que limita los alcances de estos resultados, sugiere la existencia de una relación entre obstrucción al flujo aéreo y el consumo de cantidades mayores de marihuana.

Un estudio muy reciente incluyó la revisión de datos de 7.716 sujetos pertenecientes a la NAHNES (National Health and Nutrition Examination Survey) III, mostrando que la exposición acumulada a más de 20 "pitos/año" se asociaba con disminución en la relación $\mathrm{VEF}_{1} / \mathrm{CVF}(<70 \%)$, que era más bien explicada por el aumento en la CVF que por la disminución del $\mathrm{VEF}_{1}{ }^{64}$. El significado clínico y el mecanismo responsable del aumento de la CVF observado en este y otros estudios no están aún del todo claros. Mientras algunos autores han sugerido que se debería al "estiramiento" del pulmón producido por las inhalaciones profundas repetitivas del fumador de marihuana ${ }^{50}$, otros lo relacionan como un signo de obstrucción temprana al flujo aéreo ${ }^{65}$.

Por tanto, si bien la evidencia no es concluyente y en ausencia de estudios de mejor metodología, pareciera ser que los efectos deletéreos de la inhalación de marihuana a largo plazo dependerán de la cantidad acumulada consumida en el tiempo.

\section{b) Enfermedad Pulmonar Obstructiva Crónica (EPOC)}

Como se mencionó, la evidencia actual permite establecer que la inhalación ocasional y no acumulativa de marihuana no aumentaría significativamente el riesgo de desarrollar EPOC, no estando definido aún si un mayor nivel de consumo determina mayor riesgo. Una hipótesis especulativa para explicar esta evidencia preliminar de ausencia de efecto adverso, se ha basado en la acción antiinflamatoria del THC, lo que podría contrarrestar los efectos inflamatorios producidos por los otros componentes del humo de la marihuana ${ }^{66}$.

Un aspecto muy importante de los fumadores de marihuana es que la mayoría de ellos también fuman tabaco. Los efectos combinados de ambos sobre el pulmón también han sido estudiados. Tan y cols ${ }^{67}$, mostraron que el uso de ambas drogas se asocia con un riesgo aumentado (comparado con los fumadores de sólo cigarrillos) de desarrollar EPOC (OR: 2,9; IC $95 \%$ : 1,53-5,51) cuando la dosis acumulada durante la vida sobrepasó los 50 pitos/año, lo que sugiere la existencia de sinergia entre el consumo de marihuana y el tabaco. En este estudio no se detectó asociación entre el fumar sólo marihuana y la EPOC, aunque 
el poder estadístico para detectar la existencia de alguna asociación fue bajo.

\section{c) Síntomas respiratorios crónicos}

En la actualidad no hay dudas que fumar marihuana induce la aparición de síntomas respiratorios. Esto se ha demostrado en estudios poblacionales que han controlado en sus resultados el hábito tabáquico, los que han mostrado consistentemente mayor frecuencia de tos, expectoración y sibilancias en los fumadores regulares de marihuana versus los no fumadores ${ }^{50} \mathrm{y}$ que estos síntomas desaparecen al dejar de fumarla ${ }^{68,69}$. Es importante notar que estos estudios se han hecho en fumadores regulares de marihuana, por tanto en sujetos con dosis acumuladas significativas en el tiempo.

\section{d) Inflamación e inmunomodulación}

Existen múltiples evidencias que demuestran que el uso inhalado a largo plazo de la marihuana induce cambios inflamatorios y en el sistema inmune en la vía aérea. Por ejemplo, se ha comunicado que los macrófagos alveolares en los fumadores de marihuana, comparado con los no fumadores, muestran alteración en su capacidad bactericida, antitumoral y de secreción de citoquinas inflamatorias ${ }^{70}$. Estudios broncoscópicos han mostrado que el humo de la marihuana produce un aumento en el puntaje visual de bronquitis de magnitud similar a la producida por el tabaco y que las biopsias de estos sujetos muestran hiperplasia vascular, edema de la submucosa, infiltrados celulares inflamatorios, engrosamiento de la membrana basal e hiperplasia de las células caliciformes $^{71,72}$. Lo más llamativo es que estos cambios inflamatorios se han descrito en fumadores habituales de marihuana aparentemente sanos (relativamente asintomáticos) $^{71}$.

Probablemente la persistencia e intensidad de esta inflamación, la hiperplasia de las células caliciformes y la pérdida de células epiteliales bronquiales ciliadas es lo que explica la aparición de síntomas en los fumadores regulares de marihuana ${ }^{66}$.

En cuanto a los efectos sobre el sistema inmune, se sabe que los cannabinoides tienen acciones inmunomoduladoras por intermedio de los receptores endocanabinoides presentes en muchas vías del sistema inmune ${ }^{73}$ afectando el funcionamiento de los linfocitos B, T y las células NK (natural killer $)^{74}$. También pueden alterar la expresión de una serie de citoquinas como las interleuquinas (IL-6, IL-8, IL-10, IL-12), el factor de necrosis tumoral $(\mathrm{TNF}-\alpha)$ y el interferón- $\gamma(\mathrm{INF}-\gamma)^{75}$. El efecto sobre la actividad microbicida de los ma- crófagos, comentada más arriba, está dada por el efecto inmunosupresor del THC mediante la activación de los receptores $\mathrm{CB} 2^{50}$. Si bien no están claras las consecuencias de esta inmunomodulación, podrían resultar en una mayor predisposición a desarrollar infecciones de la vía aérea. De hecho, recientemente se ha advertido sobre los efectos de varias formas de contaminación presentes en la planta de cannabis antes de ser secada y prensada. En particular, el Aspergillus fumigatus es prevalente en las plantas de cannabis que crecen dentro de las casas ${ }^{76}$, existiendo varios reportes de infección pulmonar al fumar cannabis contaminada con el hongo ${ }^{77}$.

\section{e) Cáncer pulmonar}

En comparación con una cantidad comparable de humo de cigarrillo, el humo de la marihuana contiene cerca de $50 \%$ más benzopireno y $75 \%$ más benzantraceno, ambos hidrocarburos aromáticos policíclicos procarcinogénicos, como también otros carcinógenos y co-carcinógenos como fenoles, nitrosaminas, radicales libres, etc. ${ }^{50}$. De esta forma, el humo de la marihuana podría provocar, al menos, alteraciones histopatológicas tan extensas como el humo del cigarrillo, incluyendo cambios metaplásicos y alteraciones nucleares que podrían ser premalignas. Por lo menos esto es lo que muestran los estudios experimentales tanto en animales como en seres humanos.

Estudios en ratas expuestas a condensado de humo de marihuana y a condensado de humo de cigarrillo han mostrado que ambas exposiciones inducen cambios metabólicos asociados al desarrollo de cáncer como expresión de genes, estrés oxidativo, alteración del ADN, etc. ${ }^{78}$.

Por otra parte, en seres humanos fumadores de marihuana, se han descrito cambios histopatológicos e inmunohistológicos del tipo metaplasia escamosa bronquial y sobreexpresión de marcadores moleculares de progresión pre-tumoral, lo que sugiere que el humo de la marihuana podría ser un factor de riesgo para el desarrollo de cáncer pulmonar ${ }^{72,79,80}$.

Revisiones recientes han mostrado, en general, la existencia de una asociación entre el consumo de marihuana y el desarrollo de lesiones premalignas $^{81}$, aunque esta asociación disminuye, sin dejar de ser significativa, cuando se controla por consumo de tabaco y por cantidad consumida de marihuana ${ }^{82}$. El fumar más de 30 pitos/año se ha asociado significativamente con incidencia de cáncer pulmonar, aunque la significancia de este hallazgo se pierde cuando se controla por edad, género, raza, nivel educacional, consumo de alcohol y consumo de tabaco ${ }^{83}$. 
Uno de los problemas principales para establecer la existencia de una asociación causal es la escasez de estudios epidemiológicos que evalúan la asociación entre el hábito de fumar marihuana con el desarrollo de cáncer pulmonar ${ }^{61,83,84} \mathrm{y}$, los que existen, están sujetos a muchos factores confundentes, principalmente el consumo concomitante de tabaco. Por ejemplo, un análisis de estudios caso-control en Africa mostró que la razón de disparidad (OR) para cáncer pulmonar fue de 2,4 (IC I5\% $\left._{1}: 1,6-3,8\right)^{85}$ en fumadores de marihuana y tabaco. Uno de los estudios con mayor tiempo de seguimiento mostró que los grandes fumadores de marihuana (definido como más de 50 pitos/año) tuvieron más del doble de riesgo de desarrollar cáncer pulmonar tras más de 40 años de seguimiento ${ }^{86}$. Este estudio controló según tabaquismo, consumo de alcohol, enfermedades respiratorias y estado socio-económico basales.

\section{f) Otras alteraciones pulmonares}

Se han descrito casos de neumotórax espontáneo en los fumadores de grandes cantidades de marihuana ${ }^{87}$, sin poderse aún establecer si esta asociación se origina por la marihuana en sí o es consecuencia de la técnica de inhalación que usan los fumadores al consumirla (inhalaciones más profundas y mantención de la respiración por mayor tiempo que los fumadores de cigarrillos, lo que se acompaña de maniobras de Valsalva). Los estudios sobre este tema son escasos y los que existen presentan debilidades metodológicas para dilucidar el mecanismo (incluyendo el uso concomitante de cocaína).

Tampoco hay evidencias que apunten a atribuir al consumo de marihuana un mayor riesgo para desarrollar enfisema pulmonar ${ }^{61}$.

\section{Conclusiones}

Existe la necesidad urgente de realizar estudios metodológicamente bien diseñados con el fin de especificar el verdadero rol terapéutico de la marihuana y su perfil de seguridad a largo plazo en las diferentes patologías propuestas. Por otro lado, la evidencia muestra que el uso recreacional de la marihuana, además de efectos psicosociales deletéreos, induce sobre el sistema respiratorio la aparición de síntomas crónicos de la vía aérea, inflamación local y efectos inmunomoduladores y, dependiendo del tiempo y cantidad de exposición, efectos negativos sobre la función pulmonar, desarrollo de EPOC y cáncer pulmonar. Por tanto, debido al impacto negativo tanto personal como social que conlleva el consumo de la marihua- $\mathrm{na}^{88}$, no puede considerarse como una droga libre de riesgos para la salud cuyo consumo debiera permitirse ${ }^{89}$.

\section{Bibliografía}

1.- UNITED NATIONS OFFICE ON DRUGS AND CRIME. World Drug Report 2013. United Nations publication, Sales No. E.13.XI.6. www.unodc.org unode/ secured/wdr/wdr2013/World_Drug_Report_2013.pdf

2.- SENDA. Observatorio Chileno de Drogas. Décimo Primer Estudio Nacional de Drogas en Población General. Resultados Generales. Julio 2015. http://www.senda. gob.cl/wp-content/uploads/2015/07/Informe-EjecutivoENPG-2014.pdf

3.- www.dea.gov/druginfo/ds.shtml. (Acceso 11 de julio de 2015).

4.- HILL K P. Medical Marijuana for Treatment of Chronic Pain and Other Medical and Psychiatric Problems A Clinical Review. JAMA 2015; 313: 2474-83.

5.- VOLKOW N D, BALER R D, COMPTON W M, WEISS S R B. Adverse Health Effects of Marijuana Use. N Engl J Med 2014; 370: 2219-27.

6.- COOMBES R. Cannabis regulation: high time for change? BMJ 2014; 348: g3382.

7.- D'SOUZA D C, RANGANATHAN M. Medical Marijuana. Is the Cart Before the Horse? JAMA 2015; 313 : 2431-2.

8.- PERTWEE R G. Cannabinoid pharmacology: the first 66 years. Br J Pharmacol 2006; 147 (suppl 1): S163-71.

9.- VAN SICKLE M D, DUNCAN M, KINGSLEY P J, MOUIHATE A, URBANI P, MACKIE K, et al. Identification and functional characterization of brainstem cannabinoid CB2 receptors. Science 2005; 310: 329-32.

10.- BHATTACHARYYA S, MORRISON P D, FUSARPOLI P, MARTIN-SANTOS R, BORGWARDT S, WINTON-BROWN T, et al. Opposite effects of delta-9tetrahydrocannabinol and cannabidiol on human brain function and psychopathology. Neuropsychopharmacology 2010; 35: 764-74.

11.- BENSON M, BENTLEY A M. Lung disease induced by drug addiction. Thorax 1995; 50: 1125-7.

12.- HILL K P. Medical Marijuana for Treatment of Chronic Pain and Other Medical and Psychiatric Problems. A Clinical Review. JAMA 2015; 313: 2474-83.

13.- VANDREY R, RABER J C, RABER M E, DOUGLASS B, MILLER C, BONN-MILLER M O. Cannabinoid Dose and Label Accuracy in Edible Medical Cannabis Products. JAMA 2015; 313: 2491-3.

14.- AGGARWAL S K, KYASHNA-TOCHA M, CARTER G T. Dosing medical marijuana: rational guidelines on trial in Washington State. Med Gen Med 2007; 9: 52.

15.- CORRIGAN D. The pharmacology of cannabis: issues for understanding its use. In: A cannabis reader: global issues and local experiences. European Monitoring Centre for Drugs and Drug Addiction Monograph 2008; 
8: 30-7. www.emcdda.europa.eu/attachements.cfm/ att 53370 EN emcdda-cannabis-mon-vol1-ch3-web. pdf (acceso el 17 de septiembre de 2015).

16.- http://www.fda.gov/newsevents/publichealthfocus/ ucm421168.htm\#safe (acceso el 11 de julio de 2015).

17.- http://www.ema.europa.eu/ema/index.jsp?curl=pages/ medicines/pips/EMEA-000181-PIP01-08-M01/ pip_000125.jsp\&mid=WC0b01ac058001d129 (acceso el 11 de julio de 2015).

18.- VENEGAS V. Legalización de la Marihuana. La libertad que amenaza la salud pública. Rev Chil Pediatr 2014; 85: 653-7.

19.- MACHADO ROCHA F C, STÉFANO S C, DE CÁSSIA HAIEK R, ROSA OLIVEIRA L M Q, DA SILVEIRA D X. Therapeutic use of Cannabis sativa on chemotherapy-induced nausea and vomiting among cancer patients: systematic review and meta-analysis. Eur J Cancer Care 2008; 17: 431-43.

20.- NAVARI R M. Pharmacological Management of Chemotherapy-Induced Nausea and Vomiting. Drugs 2009; 69: 515-33

21.- FARRELL M, BUCHBINDER R, HALL W. Should doctors prescribe cannabinoids? BMJ 2014; 348: g2737.

22.- LUTGE E E, GRAY A, SIEGFRIED N. The medical use of cannabis for reducing morbidity and mortality in patients with HIV/AIDS. Cochrane Database Syst Rev 2013; 4: CD005175.

23.- WILKINSON S T, D'SOUZA D C. Problems With the Medicalization of Marijuana. JAMA 2014; 311: 2377-8.

24.- LYNCH M E, CAMPBELL F. Cannabinoids for treatment of chronic non-cancer pain; a systematic review of randomized trials. Br J Clin Pharmacol 2011; 72: 735-44.

25.- SNEDECOR S J, SUDHARSHAN L, CAPPELLERI J C, SADOSKY A, DESAI P, JALUNDHWALA Y J, et al. Systematic review and comparison of pharmacologic therapies for neuropathic pain associated with spinal cord injury. J Pain Res 2013; 6: 539-47.

26.- RINTALA D H, FIESS R N, TAN G, HOLMES S A, BRUEL B M. Effect of dronabinol on central neuropathic pain after spinal cord injury: a pilot study. Am J Phys Med Rehabil 2010; 89: 840-8.

27.- PHILLIPS T J, CHERRY C L, COX S, MARSHALL S J, RICE A S. Pharmacological treatment of painful HIVassociated sensory neuropathy: a systematic review and meta-analysis of randomised controlled trials. PLoS One 2010; 5: e14433.

28.- SELVARAJAH D, GANDHI R, EMERY C J, TESFAYE S. Randomized placebo-controlled double-blind clinical trial of cannabis-based medicinal product (Sativex) in painful diabetic neuropathy: depression is a major confounding factor. Diabetes Care 2010; 33: 128-30.

29.- TOTH C, MAWANI S, BRADY S, CHAN C, LIU C, MEHINA E, et al. An enriched-enrolment, randomized withdrawal, flexible-dose, double-blind, placebo-controlled, parallel assignment efficacy study of nabilone as adjuvant in the treatment of diabetic peripheral neuropathic pain. Pain 2012; 153: 2073-82.

30.- RICHARDS B, WHITTLE S, BUCHBINDER R. Neuromodulators for pain management in rheumatoid arthritis. Cochrane Database Syst Rev 2011; 1: CD008921.

31.- DE SOUZA NASCIMENTO S, DESANTANA J M, NAMPO F K, RIBEIRO E A N, DA SILVA D L, ARAUJO-JUNIOR J X, et al. Efficacy and safety of medicinal plants or related natural products for fibromyalgia: a systematic review. Evid Based Complement Alternat Med 2013; 2013: 149-468.

32.- WARE M A, FITZCHARLES M A, JOSEPH L, SHIR Y. The effects of nabilone on sleep in fibromyalgia: results of a randomized controlled trial. Anesth Analges 2010; 110: 604-10

33.- SKRABEK R Q, GALIMOVA L, ETHANS K, PERRY D. Nabilone for the treatment of pain in fibromyalgia. $J$ Pain 2008; 9: 164-73.

34.- PINI L A, GUERZONI S, CAINAZZO M M, FERRARI A, SARCHIELLI P, TIRAFERRI I, et al. Nabilone for the treatment of medication overuse headache: results of a preliminary double-blind, active-controlled, randomized trial. J Headache Pain 2012; 13: 677-84.

35.- MARTIN-SÁNCHEZ E, FURUKAWA T A, TAYLOR J, MARTIN J L. Systematic review and meta-analysis of cannabis treatment for chronic pain. Pain Med 2009; 10: 1353-68.

36.- PORTENOY R K, GANAE-MOTAN E D, ALLENDE S, YANAGIHARA R, SHAIOVA L, WEINSTEIN S, et al. Nabiximols for opioid-treated cancer patients with poorly-controlled chronic pain: a randomized, placebocontrolled, graded-dose trial. J Pain 2012; 13: 438-49.

37.- JOHNSON J R, BURNELL-NUGENT M, LOSSIGNOL D, GANAE-MOTAN E D, POTTS R, FALLON M T. Multicenter, double-blind, randomized, placebocontrolled, parallel-group study of the efficacy, safety, and tolerability of THC:CBD extract and THC extract in patients with intractable cancer-related pain. J Pain Symptom Manage 2010; 39: 167-79.

38.- FERGUSSON D M, BODEN J M. Cannabis Use and Later Life Outcomes. Addiction 2008; 103: 969-76.

39.- ASHTON C H. Pharmacology and effects of cannabis: a brief review. Br J Psychiatry 2001; 178: 101-6.

40.- HALL W, DEGENHARDT L. Adverse health effects of non-medical cannabis use. Lancet 2009; 374: 1383-91.

41.- MACCARRONE M, GUZMÁN M, MACKIE K, DOHERTY P, HARKANY T. Programming of neural cells by (endo) cannabinoids. Nat Rev Neurosci 2014; 15 : 786-801.

42.- GOGTAY N, GIEDD J N, LUSK L, HAYASHI K M, GREENSTEIN D, VAITUZIS A C, et al. Dynamic mapping of human cortical development during childhood through early adulthood. Proc Natl Acad Sci USA 2004; 101: 8174-9.

43.- MEIER M H, CASPI A, AMBLER A, HARRINGTON H, HOUTS R, KEEFE R S, et al. Persistent cannabis 
users show neuropsy- chological decline from childhood to mid- life. Proc Natl Acad Sci USA 2012;109 (40): E2657-4.

44.- LYNSKEY M, HALL W. The effects of adolescent cannabis use on educational attainment: a review. Addiction 2000; 95: 1621-30.

45.- CASPI A, MOFFITT T E, CANNON M, MCCLAY J, MURRAY R, HARRINGTON H, et al. Moderation of the effect of adolescent-onset cannabis use on adult psychosis by a functional polymorphism in the catecholO-methyltransferase gene: longitudinal evidence of a gene X environment interaction. Biol Psychiatry 2005; 57: 1117-27.

46.- BRADY J E, LI G. Trends in alcohol and other drugs detected in fatally injured drivers in the United States, 1999-2010. Am J Epidemiol 2014; 179: 692-9.

47.- LENNÉ M G, DIETZE P M, TRIGGS T J, WALMSLEY S, MURPHY B, REDMAN J R. The effects of cannabis and alcohol on simulated arterial driving: influences of driving experience and task demand. Accid Anal Prev 2010; 42: 859-66.

48.- THOMAS G, KLONER R A, REZKALLA S. Adverse cardiovascular, cerebrovascular, and peripheral vascular effects of marijuana inhalation: what cardiologists need to know. Am J Cardiol 2014; 113 (1): 187-90.

49.- MOIR D, RICKERT W S, LEVASSEUR G, LAROSE Y, MAERTENS R, WHITE $P$, et al. A comparison of mainstream and sidestream marijuana and tobacco cigarette smoke produced under two machine smoking conditions. Chem Res Toxicol 2008; 21: 494-502.

50.- TASHKIN D P. Effects of Marijuana Smoking on the Lung. Ann Am Thorac Soc 2013; 10: 239-47.

51.- TASHKIN D P, GLIEDERER F, ROSE J, CHANG P, HUI K K, YU J L, et al. Tar, CO and delta 9THC delivery from the 1st and 2nd halves of a marijuana cigarette. Pharmacol Biochem Behav 1991; 40: 657-61.

52.- LEE M H, HANCOX R J. Effects of smoking cannabis on lung function. Expert Rev Respir Med 2011; 5: 53746.

53.- AGGARWAL S K, CARTER G T, SULLIVAN M D, ZUMBRUNNEN C, MORRILL R, MAYER J D. Medicinal use of cannabis in the United States: historical perspectives, current trends, and future directions. J Opioid Manag 2009; 5: 153-68.

54.- TASHKIN D P, SHAPIRO B J, FRANK I M. Acute pulmonary physiologic effects of smoked marijuana and oral D9-tetrahydrocannabinol in healthy young men. $\mathrm{N}$ Engl J Med 1973; 289: 336-41.

55.- VACHON L, FITZGERALD M X, SOLLIDAY N H, GOULD I A, GAENSLER E A. Single-dose effect of marijuana smoke: bronchial dynamics and respiratory center sensitivity in normal subjects. N Engl J Med 1973; 288: 985-9.

56.- JOSHI M, JOSHI A, BARTTER T. Marijuana and lung diseases. Curr Opin Pulm Med 2014; 20: 173-9.

57.- TETRAULT J M, CROTHERS K, MOORE B A, ME-
HRA R, CONCATO J, FIELLIN D A. Effects of marijuana smoking on pulmonary function and respiratory complications: a systematic review. Arch Intern Med 2007; 167: 221-8.

58.- GRASSIN-DELYLE S, NALINE E, BUENESTADO A, FAISY C, ÁLVAREZ J-C, SALVATOR H, et al. Cannabinoids inhibit cholinergic contraction in human airways through prejunctional CB1 receptors. Br J Pharmacol 2014; 171: 2767-77.

59.- CALIGNANO A, KATONA I, DESARNAUD F, GIUFFRIDAA, RANA G L, MACKIE K, et al. Bidirectional control of airway responsiveness by endogenous cannabinoids. Nature 2000; 408: 96-101.

60.- UNITED NATIONS OFFICE ON DRUG AND CRIME. Cannabis. A Short Review. 2013. http://www.unodc.org/ documents/drug-prevention-and-treatment/cannabis_review.pdf (acceso el 11 de julio de 2015).

61.- ALDINGTON S, WILLIAMS M, NOWITZ M, WEATHERALL M, PRITCHARD A, MCNAUGHTON A, et al. Effects of cannabis on pulmonary structure, function and symptoms. Thorax 2007; 62: 1058-63.

62.- HANCOX R J, POULTON R, ELY M, WELCH D, TAYLOR D R, MCLACHLAN C R, et al. Effects of cannabis on lung function: a population-based cohort study. Eur Respir J 2010; 35: 42-7.

63.- PLETCHER R M J, VITTINGHOFF E, KALHAN R, RICHMAN J, SAFFORD M, SIDNEY S, et al. Association between marijuana exposure and pulmonary function over 20 years. JAMA 2012; 307: 173-81.

64.- KEMPKER J A, HONIG E G, MARTIN G S. The Effects of Marijuana Exposure on Expiratory Airflow. A Study of Adults who Participated in the US National Health and Nutrition Examination Study. Ann Am Thorac Soc 2015; 12: 135-41.

65.- BARISIONE G, CRIMI E, BARTOLINI S, SAPORITI $\mathrm{R}$, COPELLO F, PELLEGRINO R, et al. How to interpret reduced forced expiratory volume in $1 \mathrm{~s}$ (FEV1)/ vital capacity ratio with normal $\mathrm{FEV}_{1}$. Eur Respir $\mathrm{J}$ 2009; 33: 1396-402.

66.- TASHKIN D P. The respiratory health benefits of quitting cannabis use. Eur Respir J 2015; 46: 1-4.

67.- TAN W C, LO C, JONG A, XING L, FITZGERALD M J, VOLLMER W M, et al. Marijuana and chronic obstructive lung disease-a population-based study. CMAJ 2009; 180: 814-20.

68.- TASHKIN D P, SIMMONS M, TSENG C-H. Impact of changes in regular use of marijuana and/or tobacco on chronic bronchitis. COPD 2012; 9: 367-74.

69.- HANCOX R J, SHIN H H, GRAY A R, POULTON R, SEARS M R. Effects of quitting cannabis on respiratory symptoms. Eur Respir J 2015; 46: 80-7.

70.- BALDWIN G C, TASHKIN D P, BUCKLEY D M, PARK A N, DUBINETT S M, ROTH M D. Marijuana and Cocaine Impair Alveolar Macrophage Function and Cytokine Production. Am J Respir Crit Care Med 1997; 156: 1606-13. 
71.- ROTH M D, ARORA A, BARSKY S H, KLEERUP E C, SIMMONS M, TASHKIN D P. Airway Inflammation in Young Marijuana and Tobacco Smokers. Am J Respir Crit Care Med 1998; 157: 928-37.

72.- FLIGIEL S E G, ROTH M D, KLEERUP E C, BARSKIJ S H, SIMMONS M S, TASHKIN D P. Tracheobronchial Histopathology in Habitual Smokers of Cocaine, Marijuana, and/or Tobacco. Chest 1997; 112: 319-26.

73.- KLEIN T W, NEWTON C, LARSEN K, LU L, PERKINS I, NONG L, et al. The Cannabinoid System and Immune Modulation. J Leukoc Biol 2003; 74: 486-96.

74.- KLEIN T W, FRIEDMAN H, SPECTER S. Marijuana, Immunity and Infection. J Neuroimmunol 1998; 83: 102-15.

75.- CROXFORD J L, YAMAMURA T. Cannabinoids and the Immune System: Potential for the Treatment of inflammatory Diseases? J Neuroimmunol 2005; 166: 3-18.

76.- JOHNSON L I, MILLER J D. Consequences of Largescale Production of Marijuana in Residential Buildings. Indoor Built Environ 2012; 21: 595-600.

77.- GATES P, FAFFE A, COPELAND J. Cannabis smoking and respiratory health: Consideration of the literature. Respirology 2014; 19: 655-2.

78.- MAERTENS R M, WHITE P A, WILLIAMS A, YAUK C L. A global toxicogenomic analysis investigating the mechanistic differences between tobacco and marijuana smoke condensates in vitro. Toxicology 2013; 308: 6073.

79.- BARSKY S H, ROTH M D, KLEERUP E C, SIMMONS M, TASHKIN D P. Histopathologic and molecular alterations in bronchial epithelium in habitual smokers of marijuana, cocaine and/or tobacco. J Natl Cancer Inst 1998; 90: 1198-205.

80.- TASHKIN D P, BALDWIN G C, SARAFIAN T, DUBINETT S, ROTH M D. Respiratory and immunologic consequences of marijuana smoking. J Clin Pharmacol 2002; 42: 71S-81S.

81.- TASHKIN D P. Increasing cannabis use: What we still need to know about its effects on the lung. Respirology 2014; 19: 619-20.

82.- MEHRA R, MOORE B A, CROTHERS K, TETRAULT J, FIELLIN D A. The Association Between Marijuana Smoking and Lung Cancer. A Systematic Review. Arch Intern Med 2006; 166: 1359-67.

83.- HASHIBE M, MORGENSTERN H, CUI Y, TASHKIN D P, ZHANG Z F, COZEN W, et al. Marijuana Use and the Risk of Lung and Upper Aerodigestive Tract Cancers: Results of a Population-Based Case-Control Study. Cancer Epidemiol Biomarkers Prev 2006; 15: 1829-34.

84.- HASHIBE M, STRAIF K, TASHKIN D P. Epidemiologic review of marijuana use and cancer risk. Alcohol 2005; 35: 265-75.

85.- BERTHILLER J, STRAIF K, BONIOL M, VOIRIN N, BENHAÏM-LUZON V, AYOUB W B, et al. Cannabis smoking and risk of lung cancer in men: a pooled analysis of three studies in Maghreb. J Thorac Oncol 2008; 3: 1398-403.

86.- CALLAGHAN R C, ALLEBECK P, SDORCHUK A. Marijuana use and risk of lung cancer: a 40-year cohort study. Cancer Causes Control 2013; 24: 1811-20.

87.- BESHAY M, KAISER H, NIEDHART D, REYMOND M A, SCHMID R A. Emphysema and secondary pneumothorax in young adults smoking marijuana. Eur $\mathrm{J}$ Cardiothorac Surg 2007; 32: 834-8.

88.- ORGANIZACIÓN DE LOS ESTADOS AMERICANOS. El problema de las drogas en América. Capítulo 2: Drogas y Salud Pública. www.cicad.oas.org/drogas/ elinforme/informeDrogas2013/drugsPublicHealth_ESP. pdf (acceso el 20 de septiembre de 2015).

89.- MONCKEBERG F. Los pro y contra de la legalización de la marihuana. Rev Chil Pediatr 2014; 85: 229-37.

Correspondencia a:

Dr. Gonzalo Alvear T.

Clínica Miguel de Servet

Almirante Pastene 150

Providencia. Santiago de Chile.

Email:gonzaloalvear@yahoo.com 